\title{
Phase transitions in the mesoscopic superconducting square
}

\author{
${ }^{a, b}$ J.Bonča and ${ }^{a}$ V.V. Kabanov \\ ${ }^{a}$ J. Stefan Institute 1001, Ljubljana, and ${ }^{b}$ FMF, University of Ljubljana, Ljubljana, Slovenia
}

(October 25, 2018)

\begin{abstract}
We solve the Ginzburg-Landau equation (GLE) for the mesoscopic thin film of the square shape in the magnetic field. In the limit of Ginzburg-Landau parameter $\kappa \rightarrow \infty$ we find a series of first and second order phase transitions as temperature and/or magnetic field changes. First order phase transitions between giant flux states can be described with a simple variational procedure. We discuss the similarity with rotating liquid $\mathrm{He}^{4}$ and derive a simple formula for $H_{c 1}$. We identify order parameters based on symmetry arguments and we propose Landau functional describing the second order phase transition.
\end{abstract}

PACS: 74.60.Ec 74.25.Ha 74.80.-g

Advances in nanotechnology and constantly shrinking semiconductor devices have motivated researches to study properties of mesoscopic superconducting samples. One line of research in this field has focused on the problem of the phase transitions in the mesoscopic superconducting sample under the influence of the external magnetic field [1]. There are two characteristic limits in which phase transitions have different properties. If the size of the sample $a \gg \xi$, where $\xi$ is the superconducting coherence length, and applied field are large enough, there are many vortices in the sample. In this case long-range interaction between vortices and image vortices is screened by spontaneous creation of vortex loops near the sample boundary [2]. It leads to the decrease of the surface barrier for the vortex to penetrate into the sample. In the opposite case, when $a \sim \xi \ll \lambda$, with $\lambda$ being the London penetration depth, there are only few vortices in the sample. The standard Abrikosov approach [3] must be modified because of the strong influence of the sample boundaries. In this case magnetic field is almost uniform throughout the sample and long-range forces between vortices are not important. Thermodynamics of this system is determined by the short-range repulsion of vortices and Bean-Livingston barrier forces [- 4 .

Different approaches have been applied for the investigation of phase transitions in the latter limit. Most of them consider disk geometry. Buzdin and Brison applied electrostatic formalism to consider influence of the barrier on the vortex structure of the thin superconducting disk [5]. Within this approach vortices are replaced by the hard-core particles interacting through Coulomb forces and the giant vortex has never been discussed. $\mathrm{Nu}-$ merical solution of GLE for the same geometry reveals a series of the first and second order phase transitions in the superconducting disk. Such transitions take place between giant vortex states with different vorticity as well as between a giant vortex state and a multi-vortex state as the external field changes [6] 8 ]. We emphasize an important difference between the disk and the square geometry. Solution of the linearized GLE, describing the nucleation of superconducting order parameter near $H_{c 2}$ line for the disk, always corresponds to the giant vortex state. On the other hand, as it was demonstrated by Chibotaru et al. [1], there are many well separated zeros of the order parameter in the case of the square sample. Consequently, the behavior of the square sample near $H_{c 2}$ line should be qualitatively different from the disks. On the basis of the solution of the linearized GLE the appearance of the antivortex in the center of the sample has been predicted [1].

In the this paper we investigate phase transitions in the superconducting film of a square shape as a function of temperature $T$ and external magnetic field $H$ in the limit $a \sim \xi \ll \lambda$. We solve GLE for the thin superconducting film with the thickness $d \ll \xi$. We also assume that superconducting film is an extreme type two superconductor, where the Ginzburg-Landau parameter $\kappa=\lambda / \xi \gg 1$. We show that configuration with one antivortex in the center and four vortices on the diagonals of the square is unstable when we move away from the $H_{c 2}$ line and nonlinear term in the GLE is considered. On the contrary, at higher magnetic field, the configuration with four vortices on diagonals of the square remains stable. We find a sequence of phase transitions of the first order between giant vortex states as well as between multivortex states with different vorticity. Second order phase transition takes place when a giant vortex state splits into a multi-vortex state with simultaneously breaking the $C_{4}$ symmetry. Such transitions are discussed in terms of the phenomenological theory of Landau.

GLE for dimensionless complex order parameter $\psi$ has the following form:

$$
\xi^{2}\left(i \nabla+\frac{2 \pi \mathbf{A}}{\Phi_{0}}\right)^{2} \psi-\psi+\psi|\psi|^{2}=0
$$

here $\xi=\frac{\hbar^{2}}{4 m|\alpha|}, \alpha$ is temperature dependent parameter of Ginzburg-Landau expansion for the free energy, $\Phi_{0}$ is the flux quantum, $\mathbf{A}$ is the vector potential $\mathbf{H}=\nabla \times \mathbf{A}$. The second GLE equation for the vector potential can be written as: 


$$
\nabla \times \nabla \times \mathbf{A}=-i \frac{\Phi_{0}}{4 \pi \lambda^{2}}\left(\psi^{*} \nabla \psi-\psi \nabla \psi^{*}\right)-\frac{|\psi|^{2} \mathbf{A}}{\lambda^{2}} .
$$

Since we consider the case of a small mesoscopic square where $a \sim \xi \ll \lambda$, the magnetic field is uniform in the film. The correction to the external field is of the order of $1 / \kappa^{2}$ and may be found by solving Eq.(1) while assuming uniform magnetic field and substituting the solution of Eq.(1) to Eq.(2). Such a solution is equivalent to the expansion of the free energy in $1 / \kappa^{2}$ series. In addition to Eq.(1) we have to supply the boundary condition for the superconductor-insulator junction:

$$
\left(i \nabla+\frac{2 \pi \mathbf{A}}{\Phi_{0}}\right) \mathbf{n} \psi=0
$$

where $\mathbf{n}$ is normal vector to the surface of the sample.

Introducing $N \times N$ discrete points in the square we rewrite Eq.(1) in the form of nonlinear discrete Schrödinger equation:

$$
\sum_{\mathbf{l}} t_{\mathbf{i}+\mathbf{l}, \mathbf{i}} \psi_{\mathbf{i}+\mathbf{l}}-4 t_{\mathbf{i}, \mathbf{i}} \psi_{\mathbf{i}}-\psi_{\mathbf{i}}+\psi_{\mathbf{i}}\left|\psi_{\mathbf{i}}\right|^{2}=0
$$

where summation index $\mathbf{l}=( \pm 1,0),(0, \pm 1)$ points toward nearest neighbours and $t_{\mathbf{i}_{1}, \mathbf{i}}=(\xi N / a)^{2} \exp \left(-\frac{2 \pi i}{\Phi_{0}} \int_{\mathbf{i}}^{\mathbf{i}_{1}} \mathbf{A}(\mathbf{r}) d \mathbf{r}\right)$ [9]. Equivalent discretization of the boundary conditions, Eq.(3), provides an additional equation which can be directly solved and substituted into Eq.(4). As a result, equation close to the boundary are slightly different from the 'bulk':

$$
\sum_{\mathbf{l}} t_{\mathbf{i}+\mathbf{l}, \mathbf{i}} \psi_{\mathbf{i}+\mathbf{l}}-\epsilon(\mathbf{i}) t_{\mathbf{i}, \mathbf{i}} \psi_{\mathbf{i}}-\psi_{\mathbf{i}}+\psi_{\mathbf{i}}\left|\psi_{\mathbf{i}}\right|^{2}=0
$$

where $\psi_{\mathbf{i}}=0$ if $\mathbf{i}$ is outside of the sample, $\epsilon(\mathbf{i})=4-\delta_{i_{x}, 1}-$ $\delta_{i_{x}, N}-\delta_{i_{y}, 1}-\delta_{i_{y}, N}$ and $\mathbf{i}=\left(i_{x}=1, \ldots, N, i_{y}=1, \ldots, N\right)$. There is one important advantage of such a treatment of the boundary condition. When neglecting the nonlinear term in the Eq.(4), the system of linear equations reduces to the problem of eigenvalues and eigenfunction of the hermitean matrix. On the other hand, the solution of nonlinear equations requires iterations and inversion of the hermitean matrix.

Let us first discuss the solution of the linearized GLE and compare our results with previous studies [1]. The lowest eigenvalue of the linear GLE determines the upper critical field of the sample. We have calculated eigenvalues of the linear problem expressed in units $(a / \xi(T))^{2}$ as a function of the dimensionless external magnetic field $h=\Phi / \Phi_{0}$ where $\Phi$ is the total flux through the sample. Our results for a few lowest eigenvalues agree within the linewidth with the results of Ref. [1]. Spatial pattern of the order parameter is also similar. For the field $h \simeq 5.5$ we have observed 5 zeros of the order parameter near the center of the square. The solution corresponds to 4 vortices on the diagonals and one anti-vortex in the center of the square with total vorticity $m=3$ [1]. The distance

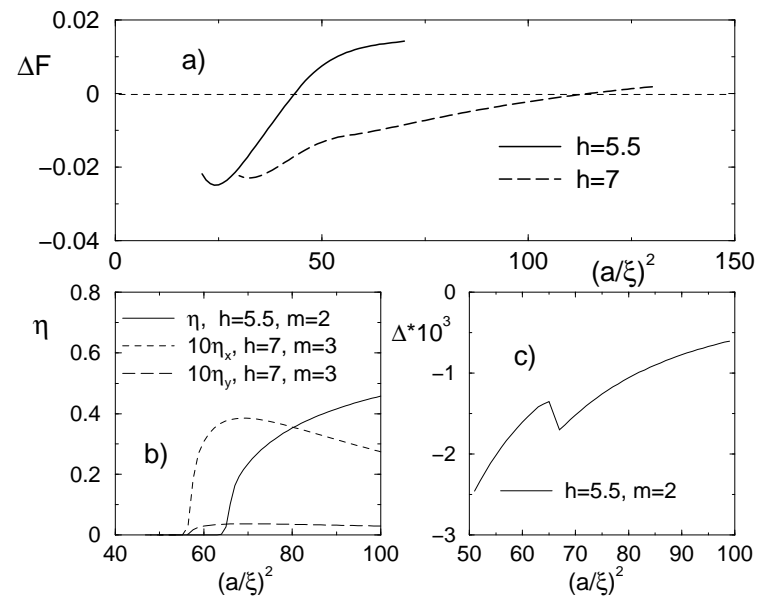

FIG. 1. a) Difference in the free energy $\Delta F$ in units of $\frac{\Phi_{0}^{2} a^{2} d}{(4 \pi)^{3} \lambda^{2} \xi^{2}}$ between solutions with different integral vorticities. For the case of $h=5.5, \Delta F=F(m=3)-F(m=2)$ and for $h=7, \Delta F=F(m=4)-F(m=3)$. b) Order parameter $\eta$ vs. $(a / \xi)^{2}$ for the solution with $m=2$ calculated at $h=5.5$ and two dimensional order parameter $\eta_{x}$ and $\eta_{y}$ for the solution with $m=3$ calculated at $h=7$. c) The second derivative of the free energy $\Delta=d^{2} F / d\left((a / \xi)^{2}\right)^{2}$ for the solution with $m=2$ at $h=5.5$.

between vortices is of the order of $\delta \simeq 0.12 \xi<<\xi$. All these zeros are pinned in the region of the square where $|\psi(x, y)|$ is small (four orders less then the value of the order parameter near the sample boundary). This indicates that vortex-anti-vortex structure becomes unstable when we move away from the $H_{c 2}$ line and nonlinear term and $1 / \kappa$ corrections are considered. Moreover, the value of the order parameter and the screening current are small near zeros. In that case correction to the external field is determined by the current flowing around all 5 zeros of the order parameter rather than by the current between them. Therefor, we do not expect suppression of the field in the core of the anti-vortex.

In the following we consider the changes in the vortex structure when we move away from the $H_{c 2}$ line. Taking into account the nonlinear term, we were unable to detect more than one zero of the order parameter near the origin of the square for the value of the field $h=5.5$. Consequently, the solution with more than one zero survives only very close to $H_{c 2}$ line. We do not expect any phase transition at the point where all zeros are joined together since total flux and the symmetry of the solution do not change. This situation is different from the case of higher field $h=7$, where solution with four zeros of the order parameter survives far from the $H_{c 2}$ line. In fact, the giant flux solution was not detected in that case.

In the Fig. (11a) we plot the difference in the free energy between solutions with different integral vorticity $\Delta F=F(m=3)-F(m=2)$ as a function of $(a / \xi)^{2}$ for 
the fixed magnetic field $h=5.5$. As it is clearly seen from the figure, near $(a / \xi)^{2} \sim 43$ a first order phase transition takes place. At that point the high-temperature phase corresponding to the giant vortex with $m=3$ becomes metastable, and the phase corresponding to giant vortex, shown in Fig. (2 top), with $m=2$ becomes a ground state. At this point, the slope of the first derivative of the free energy as a function of $T$ is discontinuous, which corresponds to the latent heat of the transition. With further decrease of the temperature and $\xi$, the second transition takes place. At $(a / \xi)^{2} \sim 66$ the giant vortex located in the center splits along one of the diagonals, Fig. (2 bottom). This transition is the second order phase transition, where $|\psi|^{2}$ is no longer invariant under four-fold axis of the square. The phase transition is clearly observed by computing the order parameter $\eta=\int x y|\psi(x, y)|^{2} d x d y$, presented in Fig. (11b). A nonvanishing $\eta$ is followed by a jump in the second derivative of the free energy $\Delta=d^{2} F / d\left((a / \xi)^{2}\right)^{2}$, presented in Fig. (11 c). We estimated the magnitude of the effect in terms of the specific heat jump: $\Delta C / a^{2} d=\frac{5 \cdot 10^{-3} \Phi_{0}^{2} T_{c}^{\prime}}{(4 \pi)^{3} \lambda^{2} \xi^{2} T_{c}^{2}}$, where $T_{c}^{\prime}$ is the critical temperature of the transition determined by the condition $(a / \xi)^{2} \simeq 66$, and $T_{c}$ is the critical temperature of the sample at $h=0$. Landau functional, describing this phase transition, is defined as $F=\alpha_{1} \eta^{2}+\beta_{1} \eta^{4}$, where $\alpha_{1}$ and $\beta_{1}$ are Landau coefficients with $\alpha_{1} \propto\left(T-T_{c}^{\prime}\right)$. This phase transition corresponds to the one dimensional corepresentation $B$ of the nonunitary $C_{4 v}\left(C_{4}\right)$ group.

Since we restrict our calculation to the limit of $\kappa \rightarrow \infty$ and $\lambda \gg a$, long range forces $(r \gg \xi)$ between vortices are irrelevant. At short distances $r \sim \xi$ there is short range repulsion between them. There is another interaction of vortices with the boundaries of the sample, known as Bean-Livingston barrier [4]. In the vicinity of $H_{c 2}$ line the interaction with the boundaries is larger than the repulsion between vortices. This is the case for the magnetic field $h=5.5$, where the giant vortex with $m=3$ is located in the center of the sample. With the decrease of $\xi$ the energy difference between two different solutions with different vorticity decreases and the first order phase transition to a giant flux state with vorticity $m=2$ takes place at $(a / \xi)^{2} \simeq 43$. A giant flux state (with lower vorticity) remains stable, since the interaction with the sample boundaries still dominates over the repulsion between vortices. Further decrease of $\xi$ leads to the decrease of the interaction of the vortices with the boundaries. BeanLivingston force decreases and repulsion of the vortices in the giant flux state starts to dominate. As a result of the interplay of the vortex-vortex repulsion and the repulsion of vortex from the boundaries, the second order phase transition $\left(\right.$ at $\left.(a / \xi)^{2} \simeq 66\right)$ between the giant vortex state and the multivortex state takes place, preserving the integral vorticity. Separation between vortices is determined by the vortex-vortex repulsion that tends to
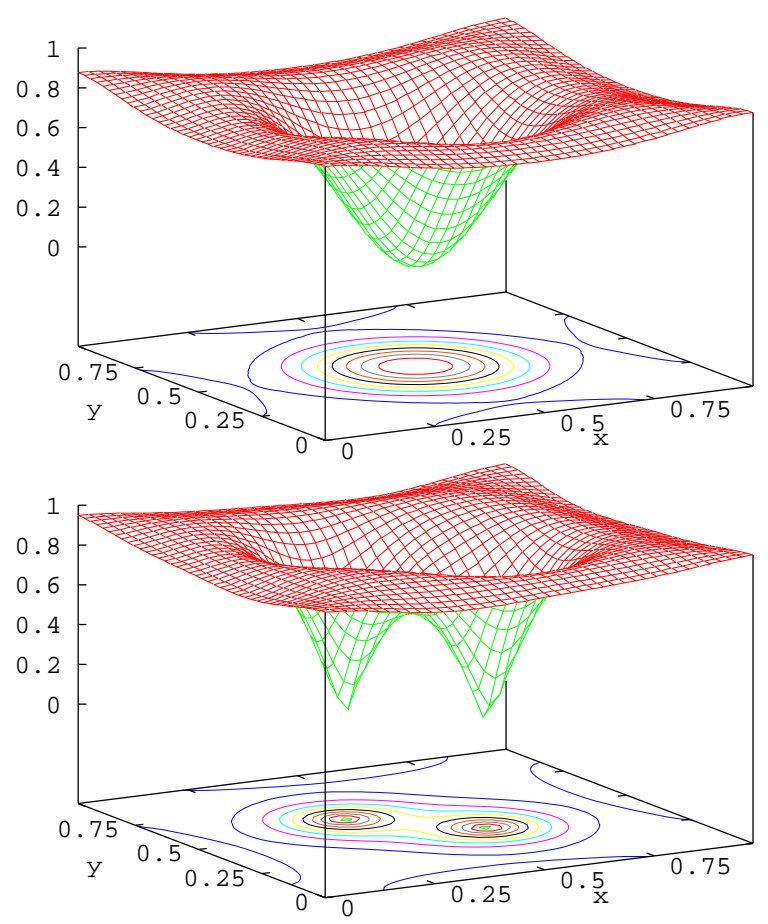

FIG. 2. $|\psi(x, y)|$ calculated at fixed magnetic field $h=5.5$. Presented are solutions with lowest free energy calculated at $(a / \xi)^{2}=50$ with one giant flux $m=2$ (top) and $(a / \xi)^{2}=90$ with two separated fluxes each carrying $m=1$ (bottom). Contours represent $|\psi(x, y)|=0.1, \ldots, 0.9$.

separate them as far as possible, while in the contrary, repulsion from the boundaries prevents vortices from approaching the boundaries.

Situation is different when external magnetic field is increased to $h \simeq 7$. In that case near $H_{c 2}$ line ground state corresponds to a multivortex state with the total vorticity $m=4$, Fig. (3 top). When temperature decreases, the first order phase transition takes place at $(a / \xi)^{2}=110$, see Fig. (19). At this point multivortex state with $m=4$ becomes unstable while the multivortex state with $m=3$, presented in Fig. (3 bottom), represents the solution with the lowest free energy. Apart from the change of the vorticity, the symmetry is also reduced at the transition point. Phase transition takes place in accordance with joint corepresentation $E^{ \pm}$of the nonunitary group $C_{4 v}\left(C_{4}\right)$. Consequently, four orientations of the pseudodipolar moment of the vortices are possible. Two component order parameter corresponding to given change of symmetry, presented in Fig. (1 $\mathrm{b}$ ), can be determined as follows: $\eta_{x}=\int x|\psi(x, y)|^{2} d x d y, \eta_{y}=\int y|\psi(x, y)|^{2} d x d y$. Free energy in that case depends on the vorticity $m$ and order parameter $\eta_{x}, \eta_{y} . \quad F\left(m=4, \eta_{x}, \eta_{y}\right)$ always has a minimum at $\eta_{x}=\eta_{y}=0$. For $m=3, F\left(m=3, \eta_{x}, \eta_{y}\right)=$ $\alpha_{2}\left(\eta_{x}^{2}+\eta_{y}^{2}\right)+\beta_{2}\left(\eta_{x}^{4}+\eta_{y}^{4}\right)+\gamma_{2} \eta_{x}^{2} \eta_{y}^{2}$, where $\alpha_{2}, \beta_{2}, \gamma_{2}$ are Landau coefficients, $\alpha_{2} \propto\left(T-T_{c}^{\prime}\right)$ where $T_{c}^{\prime}$ is the temperature of the transition between the giant vortex state 

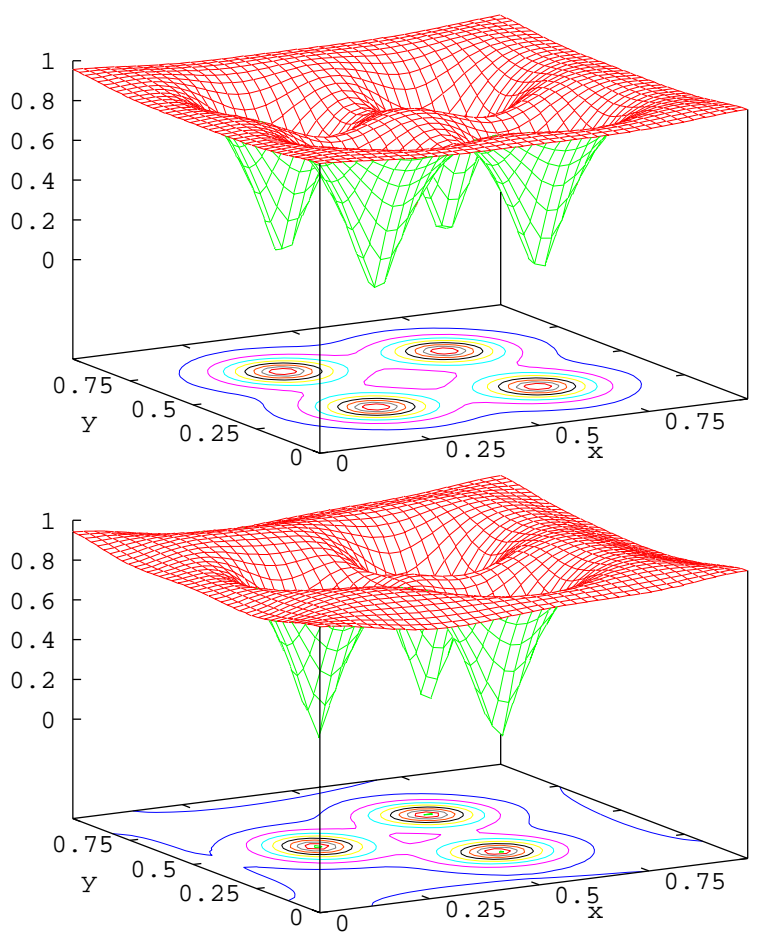

FIG. 3. $|\psi(x, y)|$ calculated at fixed magnetic field $h=7$. Presented are solutions with lowest free energy calculated at $(a / \xi)^{2}=100$ (top) and $(a / \xi)^{2}=120$ (bottom). Contours are defined as in Fig. (2).

and the multivortex state for the case of $m=3 . T_{c}^{\prime}$ is determined by the condition $(a / \xi)^{2} \simeq 56$. This transition is unobservable because $T_{c}^{\prime}$ is lower than the transition temperature for the first order phase transition where the vorticity $m$ changes form $m=3$ to $m=2$ (compare Figs. (11a) and (1/b)).

The first order phase transition between giant vortex states with different vorticity can be described qualitatively on the basis of a simple variational function for the order parameter. Spatial dependence of the order parameter in the giant vortex state with vorticity $m$ can be approximated by the function:

$$
\psi(r, \phi)=\left\{\begin{aligned}
(r / \xi)^{m} \exp (i m \phi) ; & r<\xi \\
\exp (i m \phi) ; & r>\xi
\end{aligned}\right.
$$

Substituting this function into the Ginzburg-Landau functional and keeping only leading terms in $(a / \xi)^{2}$, we obtain a simple expression for the free energy which should be minimized as a function of vorticity $m$ :

$$
F \simeq \frac{\Phi_{0}^{2} d}{(8 \pi)^{2} \lambda^{2}}\left(m^{2} \ln \left(a^{2} / \pi \xi^{2}\right) / 2-\Phi \cdot m / \Phi_{0}\right) .
$$

Minimization of the Eq.(7) in the large $m \gg 1$ limit provides the expression for the vorticity:

$$
m \simeq \frac{\Phi}{\Phi_{0}} / \ln \left(a^{2} / \pi \xi^{2}\right)
$$

Minimization of the Eq.(7) for $h=5.5$ provides that the phase transition from $m=3$ to $m=2$ takes place at $(a / \xi)^{2}=28$ which is lower than calculated value $(a / \xi)^{2}=$ 43. Nevertheless, this simple variational formula provides simple explanation of the transition with the change of vorticity in the giant vortex. It is interesting to note that due to logarithmic term, Eq.(7) for the free energy is similar to the free energy of the rotating superfluid liquid [10]. This similarity appears because $\lambda>a$ and all integrals are cut at $a$, rather than at $\lambda$. Eq.(7) yields also the estimate for $H_{c 1}$, the field at which the first vortex appears in the sample. Substituting $m=1$ to the Eq.(1) and solving equation $F(m=1)=0$ we obtain:

$$
H_{c 1}=\frac{\Phi_{0}}{2 a^{2}} \cdot \ln \left(a^{2} / \pi \xi^{2}\right) .
$$

This expression is similar to the bulk $H_{c 1}$ where $\lambda^{2}$ is substituted to $a^{2} / \pi$.

In conclusion we have solved GLE in the limit of $\kappa \rightarrow$ $\infty$ for the thin square film. We have predicted a series of the first and second order phase transitions with the change of temperature and magnetic field. On the basis of the symmetry we constructed Landau functional for the second order transitions. First order transitions are described on the basis of variational estimates.

We wish to thank D. Mihailović, V.V. Moshchalkov and A.S.Alexandrov for many useful discussions and suggestions. We are also grateful to I. Sega for critically reading the manuscript.

[1] L.F. Chibotaru, A. Ceulemans, V. Bruyndoncx, V.V. Moshchalkov Nature 408, 833 (2000).

[2] M.B. Sobnack, F.V. Kusmartsev Phys. Rev. Lett. 86, 716 (2001).

[3] A.A. Abrikosov, ZhETF, 32, 1442 (1957).

[4] P.C. Bean, J.B. Livingston, Phys. Rev. Lett., 12, 14 (1964).

[5] A.I. Buzdin, J.P. Brison, Phys. Lett. A, 196, 267 (1994).

[6] P.S. Deo, V.A. Schweigert, F.M. Peeters, and A.K. Geim, Phys. Rev. Lett. 79, 4653 (1997).

[7] V.A. Schweigert, F.M. Peeters, P.S. Deo, Phys. Rev. Lett. 81, 2783 (1998).

[8] V.A. Schweigert, F.M. Peeters, Phys. Rev. Lett. 83, 2409 (1999).

[9] Discrete nonlinear Schrödinger equation (4) is similar to the equation describing Holstein polaron on a lattice V.V. Kabanov O.Y. Mashtakov, Phys. Rev. B, 47, 6060 (1993).

[10] I.M. Khalatnikov 'Introduction to the theory of superfluidity' Nauka, Moscow, 1965; W.F. Vinen, in Superconductivity, Ed. by R.D. Parks (Marcel Dekker, New York, 1969), Vol.2, p.1167. 